\title{
SEROTYPES OF STREPTOCOCCUS PNEUMONIAE IN CHILDREN OF RUSSIAN FEDERATION
}

\author{
R. Kozlov ${ }^{1}$, A. Chagaryan ${ }^{1}$, A. Muraviov ${ }^{1}$, L. Kozlova ${ }^{1}$, E. Savin ${ }^{2}$, M. Scherbakov ${ }^{2}$, E. Shpeer ${ }^{2}$ \\ ${ }^{1}$ Smolensk State Medical Academy, Smolensk, ${ }^{2}$ GlaxoSmithKline, Moscow, Russia
}

Objective: To describe serotypes of Streptococcus pneumoniae from healthy children and paediatric patients $<5$ years old.

Study design: Multi-center microbiological and epidemiological study.

Materials and methods: 584 strains of pneumococci from 18 cities of Russian Federation were studied using PNEUMOTEST-LATEX test (Statens Serum Institut, Denmark). Among those 306 from healthy carriers, 14 from patients with invasive pneumococcal disease (IPD), 262 from non-invasive pneumococcal disease (NIPD). Population included children of day-care centers (163), orphanages (143) and paediatric hospitals (278).

Results: In children of day-care centers predominant serotypes were as follows: 19 (28.8\%), $6(15.9 \%), 15$ $(10.4 \%), 23(9.2 \%), 3(6.7 \%)$ and $9(6.7 \%)$. In orphanages serotype 19 was predominant $(67.8 \%)$, followed by $6(9.8 \%), 23(9.2 \%), 14(4.2 \%), 18(2.1 \%)$. In $1.2 \%$ of cases serotype was not identified. Ten-valent vaccine coverage for healthy carriers was $80.7 \%$. In paediatric patients with AOM serotype 19 was detected in 31.0\%, 3 (10.2\%), 14 (7.7\%), 15 (5.1\%), 23 (5.1\%), 4 (5.1\%), 6 (5.1\%), 8 (2.5\%), 9 (2.5\%), 20 (2.5\%), 22 (2.5\%). Serotypes not included in vaccine composition, were detected in $20.7 \%$. Vaccine coverage for AOM was $56.5 \%$. In CAP patients, serotype 19 was detected in 33.6\%, $6(15.8 \%), 23(8.9 \%)$ and $14(7.2 \%)$. Vaccine coverage for CAP was $70.6 \%$. Among strains from IPD, $50 \%$ were of 19 serotype.

Conclusion: An average vaccine coverage in this study was $75.2 \%$. All penicillin-resistant strains were covered by 10 -valent vaccine. 